\title{
HYPERBOLICITY THROUGH STABLE SHADOWING FOR GENERIC GEODESIC FLOWS
}

\author{
MÁRIO BESSA, JOÃO LOPES DIAS, AND MARIA JOANA TORRES
}

\begin{abstract}
We prove that the closure of the closed orbits of a generic geodesic flow on a closed Riemannian $n \geq 2$ dimensional manifold is a uniformly hyperbolic set if the shadowing property holds $C^{2}$-robustly on the metric. We obtain analogous results using weak specification and the shadowing property allowing bounded time reparametrization.
\end{abstract}

Keywords: geodesic flow, hyperbolic sets, shadowing, specification. 2010 Mathematics Subject Classification: Primary: 53D25, Secondary: 37D30.

\section{INTRODUCTION}

A major challenge in the numerical study of dynamical systems is the control of the quality of the approximation. The numerical results can become irrelevant if errors are propagated and solutions are not related to orbits of the system. It is therefore important to determine the conditions that avoid such outcome. There are two properties that are of great relevance in this context.

The shadowing property consists in the approximation of orbits affected with a certain error by true orbits.

The weak specification property, introduced by Bowen [7], can be summarized in the following way: any two pieces of orbits can be $\varepsilon$-approximated by a periodic orbit as long as the times in between the pieces are larger than a universal constant depending only on $\varepsilon$ (cf. [10, page 193]). This concept appeared in the study of ergodic theory related to statistical mechanics.

It is well-known that among uniformly hyperbolic systems we have the shadowing and the weak specification properties. On the other hand, if one requires the stability of shadowing (or the stability of weak specification) property under perturbations, then uniform hyperbolicity is achieved. This has been proved recently for Hamiltonian systems (see [5] and references therein).

In the present paper we are interested in the case of geodesic flows. The projection of the orbits of the flow into the manifold are the geodesics and there is a one-to-one correspondence between closed orbits and closed geodesics. The case of stability of shadowing, weak shadowing and weak specification for 2-dimensional closed manifolds is dealt in [6]. For geodesic flows on surfaces, invariant KAM tori around elliptic orbits split the phase space and spoil the possibility of having those properties. This is not the case in higher dimensions as KAM tori are not hypersurfaces in energy level sets. Therefore, one has to improve the arguments in [6] to obtain a multidimensional result.

In this work we extend the results for surfaces to any closed Riemannian manifold, showing that generically the robustness of shadowing, of weak specification or of the shadowing property allowing bounded time reparametrization, implies the hyperbolicity of the closure of the closed orbits (Theorem 1 and Corollary 1.1). The proofs rely on the outstanding advances in the dynamics of geodesic flows made by Contreras [8] and a new idea of combining arguments with flavor of both hyperbolic dynamics (expansion, contraction and transversal intersection of stable/unstable manifolds) and elliptic dynamics (twist maps, KAM tori and Birkhoff regions). 
Notice that we are only able to show the result for a residual set of metrics, in contrast to the 2-dimensional case.

Perturbing in the class of geodesic flows differs considerably from general Hamiltonians since perturbations of the metric are not local in phase space. The Hamiltonian case [5] also uses other perturbation techniques such as the pasting lemma and the suspension theorem for which presently there are no geodesic flows versions. Moreover, there is no available $C^{1}$-closing lemma for geodesic flows, i.e. $C^{2}$ on the metric (cf. [12] for a closing lemma for $C^{1}$ metrics). This means that there are no tools to extend hyperbolicity of the closure of closed orbits to the all phase space.

The first result relating the $C^{2}$-robustness of a certain property with hyperbolicity appeared in [14]. It consists on the proof that for $C^{2}$-stable expansive geodesic flows the closure of the closed orbits is hyperbolic. More recently, Contreras [8] showed that $C^{2}$-robustness of the property of having all closed orbits hyperbolic implies hyperbolicity.

1.1. Statement of the results. In section 2 we introduce the geodesic flow and the Poincaré section map. It includes also the definitions of shadowing, shadowing with bounded reparametrization, weak specification property and topological stability.

The set of $C^{\infty}$ Riemannian metrics on a closed connected smooth manifold $M$ of dimension $n \geq 2$ is denoted by $\mathcal{R}^{\infty}$. Define $\operatorname{Per}(g)$ to be the set of closed orbits for the geodesic flow on the unit tangent bundle of a given $g \in \mathcal{R}^{\infty}$.

Consider now the $C^{2}$-interior of the set of metrics in $\mathcal{R}^{\infty}$ such that all closed orbits are hyperbolic (see definition in $\S 2.2$ ). It is denoted by $\mathscr{F}^{2}$ and its elements are usually referred to as Mañé star systems.

Theorem 1. If the geodesic flow of $g \in \mathcal{R}^{\infty}$ satisfies any of the following properties:

(A) is stably shadowable;

(B) has the stable weak specification property;

(C) is stably shadowable with bounded reparametrization;

then $g \in \mathscr{F}^{2}$.

The proof of the above theorem is contained in section 3. Notice that since (A) implies (C) once we prove Theorem 1 (C) we obtain directly Theorem 1 (A). In any case we have included a proof of Theorem 1 (A) without the need of introducing the notion of shadowing with bounded reparametrization.

Corollary 1.1. There is a set $\mathcal{G}_{1} \subset \mathcal{R}^{2}$ where $\mathcal{G}_{1}$ is $C^{2}$-open in $\mathcal{R}^{2}$ and $\mathcal{G}_{1} \cap \mathcal{R}^{\infty}$ is $C^{\infty}$-dense in $\mathcal{R}^{\infty}$ such that if $g \in \mathcal{G}_{1}$ and the geodesic flow satisfies any of the properties:
(A) is stably shadowable;
(B) has the stable weak specification property;
(C) is stably shadowable with bounded reparametrization;

then $\overline{\operatorname{Per}(g)}$ is uniformly hyperbolic.

The above corollary is a direct application of our Theorem 1 together with a result by Contreras [8, Theorem E] (see Theorem 2.5) stating the uniform hyperbolicity of the closure of the closed orbits for a metric in $\mathcal{G}_{1} \cap \mathscr{F}^{2}$.

Notice that topological stability implies the shadowing property [17]. So, the above results are also achieved by considering the robust topological stability property.

\section{Preliminaries}

In this section we introduce the setting and some essential tools available in the literature. 
2.1. Geodesic flow and Poincaré map. Let $\mathcal{R}^{r}, 2 \leq r \leq \infty$, be the set of $C^{r}$ Riemannian metrics on a closed connected smooth manifold $M$ of dimension $n \geq 2$.

The geodesic flow $\varphi_{g}^{t}$ of $g \in \mathcal{R}^{r}$ is defined on the unit tangent bundle

$$
S_{g} M=\left\{(x, v) \in T M: g_{x}(v, v)=1\right\},
$$

which is also compact. We shall denote by $d(\cdot, \cdot)$ the distance function in $S_{g} M$. The transversal Poincaré flow of $g$ at $(x, v) \in S_{g} M$ is a family of $C^{1}$-symplectomorphisms

$$
P_{g}^{t}: \Sigma_{0} \cap U_{t} \rightarrow \Sigma_{t}, \quad P_{g}^{t}(y, u)=\varphi_{g}^{\Theta(y, u, t)}(y, u)
$$

with

$$
\Theta(y, u, t)=\min \left\{s \geq 0: \varphi_{g}^{s}(y, u) \in \Sigma_{t}\right\}
$$

and $\Sigma_{t}$ is a $C^{1}$-family of transversals to the flow at $\varphi_{g}^{t}(x, v), t \geq 0$. We assume that $U_{t}$ is a sufficiently small neighbourhood of $(x, v)$ for each $t$ such that, by the implicit function theorem, $\Theta$ is $C^{1}$ and $\Theta\left(U_{t}, t\right)$ is bounded for a fixed $t>0$.

Whenever $(x, v)$ is periodic of period $\ell>0$ we say that the symplectomorphism

$$
P_{g}:=P_{g}^{\ell}
$$

is the Poincaré map on the Poincaré section $\Sigma:=\Sigma_{0} \cap U_{\ell}$. Its derivative at $(x, v)$ is the linearized Poincaré map $D P_{g}(x, v)$ on $T_{(x, v)} \Sigma$.

A closed orbit is the orbit of a periodic point.

2.2. Hyperbolic and $q$-elliptic closed orbits. A periodic point $(x, v)$ is degenerate if the associated linearized Poincaré map has an eigenvalue which is a root of unity (notice that the eigenvalues are independent of the choice of the transversal and of the point in the closed orbit).

A periodic point $(x, v)$ is called hyperbolic if the associated linearized Poincare map has all eigenvalues $|\lambda| \neq 1$. It is elliptic if it is non-hyperbolic and non-degenerate.

We define the strong stable manifold of $(x, v) \in S_{g} M$ as

$$
W^{s s}(x, v):=\left\{(\tilde{x}, \tilde{v}) \in S_{g} M: \lim _{t \rightarrow+\infty} d\left(\varphi_{g}^{t}(\tilde{x}, \tilde{v}), \varphi_{g}^{t}(x, v)\right)=0\right\}
$$

and the stable manifold of the orbit

$$
W^{s}\left(\gamma_{(x, v)}\right):=\bigcup_{t \in \mathbb{R}} W^{s s}\left(\varphi_{g}^{t}(x, v)\right),
$$

where $\gamma_{(x, v)}$ stands for the orbit of $(x, v)=\gamma_{0}$. For small $\epsilon>0$, the local strong stable manifold is an embedded disk contained in the global stable manifold $W^{s s}(x, v)$ and is defined as

$$
W_{\epsilon}^{s s}(x, v):=\left\{(\tilde{x}, \tilde{v}) \in S_{g} M: d\left(\varphi_{g}^{t}(\tilde{x}, \tilde{v}), \varphi_{g}^{t}(x, v)\right)<\epsilon \text { if } t \geq 0\right\} .
$$

By the stable manifold theorem, there exists an $\epsilon=\epsilon(x, v)>0$ such that

$$
W^{s s}(x, v)=\bigcup_{t \geq 0} \varphi_{g}^{-t}\left(W_{\epsilon}^{s s}\left(\varphi_{g}^{t}(x, v)\right)\right) .
$$

Analogous definitions hold for unstable manifolds.

If a periodic point is elliptic and has precisely $2 q$ eigenvalues with $|\lambda|=1$, the point is said to be $q$-elliptic, where $1 \leq q \leq n-1$. A $q$-elliptic closed orbit is the orbit of a $q$-elliptic periodic point. A partially hyperbolic closed orbit is a $q$-elliptic closed orbit with $q<n-1$, and a totally elliptic corresponds to $q=n-1$.

A heteroclinic point is a point in the intersection $W^{s}\left(\gamma_{1}\right) \cap W^{u}\left(\gamma_{2}\right)$ of two hyperbolic (or partially hyperbolic) closed orbits $\gamma_{1}$ and $\gamma_{2}$. 
Let $(x, v) \in S_{g} M$ be a $q$-elliptic point and $P_{g}$ the associated Poincaré map on a transversal $\Sigma$. Then $T_{(x, v)} \Sigma=E^{s} \oplus E^{u} \oplus E^{c}$ is the decomposition into stable, unstable and center subspaces invariant under $D P_{g}(x, v)$. The restriction of the linearized Poincaré map to $E^{s}$ corresponds to the eigenvalues $|\lambda|<1$, the restriction to $E^{u}$ to the ones $|\lambda|>1$ and the remaining $2 q$ correspond to $E^{c}$ with $|\lambda|=1$.

The local strong stable $W^{s s}$, unstable $W^{s u}$ and center $W^{s c}$ manifolds are local embeddings (dimensions $n-q-1, n-q-1$ and $2 q$, respectively) which are invariant under the Poincaré map, and whose tangent spaces are the $E^{s}, E^{u}$ and $E^{c}$, respectively. Moreover, the Poincaré map restricted to the center manifold $\left.P_{g}\right|_{W^{s c}}$ is locally a symplectomorphism.

We refer to [6, section 2.1] for more details and basic definitions concerning dynamical properties of the above flows.

2.3. Generic properties. As a consequence of the bumpy metric theorem [1] (cf. [9, section 2], [11, Theorem 2]), generically all periodic points are non-degenerate and the following holds.

Proposition 2.1. There is a $C^{r}$-residual set $\mathcal{G} \subset \mathcal{R}^{r}, 2 \leq r \leq \infty$, such that if $g \in \mathcal{G}$, the closed orbits of $\varphi_{g}^{t}$ with period less than a fixed bound are finite and are partially hyperbolic, hyperbolic or totally elliptic.

The following lemma is a result by Contreras [8, section 3 and Theorem 4.1] (based on techniques developed by Arnaud and Herman [3]).

Lemma 2.2. There is a $C^{\infty}$-residual set $\mathcal{G}_{0} \subset \mathcal{R}^{2}$ such that if $g \in \mathcal{G}_{0}$ and $\gamma$ is a q-elliptic closed orbit for $\varphi_{g}^{t}, q \geq 2$, then there exists a 1-elliptic closed orbit $\tilde{\gamma}$ near $\gamma$ whose Poincaré map $P_{g}$ on the 2-dimensional central manifold $W^{s c}$ is an area-preserving twist map.

For $g \in \mathcal{R}^{2}, \varepsilon>0$ and $D \subset M$, consider the set

$$
B_{\varepsilon}(g, D)=\left\{g^{\prime} \in \mathcal{R}^{2}:\left\|g-g^{\prime}\right\|_{C^{2}}<\varepsilon, g^{\prime}=g \text { on } D\right\} .
$$

We want to realize perturbations by metrics in $B_{\varepsilon}(g, \gamma)$ for some orbit $\gamma$ of the geodesic flow of $g$. So we fix transversals $\Sigma$ and $\Sigma^{\prime}$ at $p$ and $p^{\prime}$, respectively, both on $\gamma$ and consider the associated transversal Poincaré flow $P_{g}^{t}$.

Take the set $\mathcal{G}_{1} \subset \mathcal{R}^{2}$ as defined in [8]. This set is $C^{2}$-open in $\mathcal{R}^{2}$ and $\mathcal{G}_{1} \cap \mathcal{R}^{\infty}$ is $C^{\infty}$-dense in $\mathcal{R}^{\infty}$. Denote by $\operatorname{Sp}(2 n, \mathbb{R})$ the set of symplectic linear maps in $\mathbb{R}^{2 n}$.

Theorem 2.3 ([8, 15]). Let $g \in \mathcal{G}_{1} \cap \mathcal{R}^{4}$ and $\varepsilon>0$. There exists $\delta>0$ such that for any simple geodesic segment $\gamma \subset T^{*} M$ of length 1 , any linear map $\delta$-close to $D P_{g}$ in $\operatorname{Sp}(2 n-2, \mathbb{R})$ is realizable as $D P_{\hat{g}}$ for some $\hat{g} \in B_{\varepsilon}(g, \gamma)$. Moreover, for every tubular neighborhood $W$ of $\gamma$ and any finite set $\mathcal{T}$ of transverse geodesics, the support of $\hat{g}-g$ can be contained in $W \backslash V$ for some small neighborhood of the transverse geodesics $\mathcal{T}$.

The above theorem is the version of Franks' lemma for geodesic flows in any dimension manifolds. While its two-dimensional counterpart holds for all smooth metrics [9], for $n \geq 3$ dimensional manifolds it is proved only for a residual set $\mathcal{G}_{1}$.

Given a simple closed curve $\gamma \in T M$, we define the set $\mathcal{R}_{\gamma}^{\infty}$ containing all $C^{\infty}$ metrics that have $\gamma$ as an orbit of $\varphi_{g}^{t}$.

Endow this set with the $C^{2}$-topology and let

$$
B_{\varepsilon, \gamma}(g, D)=\left\{g^{\prime} \in \mathcal{R}_{\gamma}^{\infty}:\left\|g^{\prime}-g\right\|_{C^{2}}<\varepsilon, g=g^{\prime} \text { on } D\right\} .
$$

Moreover, for any $g \in \mathcal{R}_{\gamma}^{\infty}$ consider the map

$$
T_{\gamma}:\left.g \mapsto \operatorname{tr} D P_{g}\right|_{\gamma}
$$


that gives the trace of the linearized Poincaré map at $\gamma$. Below we also use the notation $B_{\delta}(a)=\{y \in \mathbb{R}:|y-a|<\delta\}$, and $\pi: T M \rightarrow M$ the canonical projection.

Lemma 2.4. Let $g \in \mathcal{G}_{1} \cap \mathcal{R}^{\infty}, \varepsilon>0$ and $\gamma$ a closed orbit for $\varphi_{g}^{t}$. Then, there is $\delta>0$ such that for any tubular neighbourhood $W \subset M$ of $\pi \gamma$,

$$
B_{\delta}\left(T_{\gamma}(g)\right) \subset T_{\gamma}\left(B_{\varepsilon, \gamma}(g, D)\right)
$$

where $D=(M \backslash W) \cup \pi \gamma$.

Proof. This follows from Theorem 2.3.

Finally, we include a result that states the uniform hyperbolicity of the closure of the set of closed orbits for generic Mañé star systems.

Theorem 2.5. ([8, Theorem E]) There is a set $\mathcal{G}_{1} \subset \mathcal{R}^{2}$ where $\mathcal{G}_{1}$ is $C^{2}$-open in $\mathcal{R}^{2}$ and $\mathcal{G}_{1} \cap \mathcal{R}^{\infty}$ is $C^{\infty}$-dense in $\mathcal{R}^{\infty}$ such that if $g \in \mathcal{G}_{1} \cap \mathscr{F}^{2}$, then $\overline{\operatorname{Per}(g)}$ is uniformly hyperbolic.

2.4. The shadowing property. Fix $\delta, T>0$. A pair of sequences $\left[\left(x_{i}, v_{i}\right),\left(t_{i}\right)\right]_{i \in \mathbb{Z}}$, where $\left(x_{i}, v_{i}\right) \in S_{g} M, t_{i} \in \mathbb{R}, t_{i} \geq T$, is a $(\delta, T)$-pseudo-orbit of $\varphi_{g}^{t}$ if

$$
d\left(\varphi_{g}^{t_{i}}\left(x_{i}, v_{i}\right),\left(x_{i+1}, v_{i+1}\right)\right)<\delta, \quad i \in \mathbb{Z} .
$$

Write

For $t \in \mathbb{R}$, take

$$
\varsigma(n)= \begin{cases}t_{0}+t_{1}+\ldots+t_{n-1}, & n>0 \\ -\left(t_{n}+\ldots+t_{-2}+t_{-1}\right), & n<0 \\ 0, & n=0\end{cases}
$$

$$
\left(x_{0}, v_{0}\right) \star t=\varphi_{g}^{t-\varsigma(i)}\left(x_{i}, v_{i}\right), \quad \varsigma(i) \leq t<\varsigma(i+1) .
$$

Denote by Rep the set of all increasing homemorphisms $\tau: \mathbb{R} \rightarrow \mathbb{R}$ such that $\tau(0)=0$, called (time) reparameterizations. For each $\varepsilon>0$ let

$$
\operatorname{Rep}(\varepsilon)=\left\{\tau \in \operatorname{Rep}:\left|\frac{\tau(t)-\tau(s)}{t-s}-1\right|<\varepsilon, s, t \in \mathbb{R}\right\},
$$

of the reparameterizations $\varepsilon$-close to the identity.

A $(\delta, T)$-pseudo-orbit $\left[\left(x_{i}, v_{i}\right),\left(t_{i}\right)\right]_{i \in \mathbb{Z}}$ is $\varepsilon$-shadowed by some orbit of $\varphi_{g}^{t}$ if there is $(\tilde{x}, \tilde{v}) \in$ $S_{g} M$ and a reparameterization $\tau \in \operatorname{Rep}(\varepsilon)$ such that

$$
d\left(\varphi_{g}^{\tau(t)}(\tilde{x}, \tilde{v}),\left(x_{0}, v_{0}\right) \star t\right)<\varepsilon, \quad t \in \mathbb{R} .
$$

The geodesic flow of $g$ is said to have the shadowing property if for any $\varepsilon>0$ there exist $\delta, T>0$ such that any $(\delta, T)$-pseudo-orbit $\left[\left(x_{i}, v_{i}\right),\left(t_{i}\right)\right]_{i \in \mathbb{Z}}$ is $\varepsilon$-shadowed by some orbit of $\varphi_{g}^{t}$.

Finally, the geodesic flow of $g$ is stably shadowable if there exists a $C^{2}$-neighborhood $\mathcal{V} \subset \mathcal{R}^{\infty}$ of $g$ such that $\varphi_{\hat{g}}^{t}$ for any $\hat{g} \in \mathcal{V}$ has the shadowing property.

2.5. Shadowing with bounded reparametrization. Shadowing with bounded reparametrization corresponds to the use of reparametrizations far from the identity but still bounded.

Given any $L>0$, define

$$
\operatorname{Rep}^{\prime}(L)=\{\tau \in \operatorname{Rep}:|\tau(t)|<L, t \in \mathbb{R}\} .
$$

A $(\delta, T)$-pseudo-orbit $\left[\left(x_{i}, v_{i}\right),\left(t_{i}\right)\right]_{i \in \mathbb{Z}}$ is $\varepsilon$-shadowed with bounded reparametrization by an orbit of $\varphi_{g}^{t}$ if there is $L>0,(\tilde{x}, \tilde{v}) \in S_{g} M$ and a reparametrization $\tau \in \operatorname{Rep}^{\prime}(L)$ such that

$$
d\left(\varphi_{g}^{\tau(t)}(\tilde{x}, \tilde{v}),\left(x_{0}, v_{0}\right) \star t\right)<\varepsilon, \quad t \in \mathbb{R} .
$$


The geodesic flow of $g$ is said to have the shadowing with bounded reparametrization property if for any $\varepsilon>0$ there exist $\delta, T>0$ such that any $(\delta, T)$-pseudo-orbit $\left[\left(x_{i}, v_{i}\right),\left(t_{i}\right)\right]_{i \in \mathbb{Z}}$ is $\varepsilon$-shadowed with bounded reparametrization by some orbit of $\varphi_{g}^{t}$.

Finally, we say that the geodesic flow of $g$ is stably shadowable with bounded reparametrization if there exists a $C^{2}$-neighborhood $\mathcal{V} \subset \mathcal{R}^{\infty}$ of $g$ such that $\varphi_{\hat{g}}^{t}$ for any $\hat{g} \in \mathcal{V}$ has the shadowing with bounded reparametrization property.

2.6. The specification property. Consider a $\varphi_{g}^{t}$-invariant compact set $\Lambda \subset S_{g} M$. A specification $\mathcal{S}=(\sigma, P)$ consists in a finite collection $\sigma=\left\{I_{1}, \ldots, I_{m}\right\}$ of bounded disjoint intervals $I_{i}=\left[a_{i}, b_{i}\right]$ of the real line and a map $P: \bigcup_{I_{i} \in \sigma} I_{i} \rightarrow \Lambda$ such that for any $t_{1}, t_{2} \in I_{i}$ we have

$$
\varphi_{g}^{t_{2}}\left(P\left(t_{1}\right)\right)=\varphi_{g}^{t_{1}}\left(P\left(t_{2}\right)\right) \text {. }
$$

The specification $\mathcal{S}$ is $K$-spaced if $a_{i+1} \geq b_{i}+K$ for all $i \in\{1, \cdots, m\}$ and the minimal of such $K$ is called the spacing of $\mathcal{S}$. If $\sigma=\left\{I_{1}, I_{2}\right\}$, then $\mathcal{S}$ is said to be a weak specification. Given $\varepsilon>0$, we say that $\mathcal{S}$ is $\varepsilon$ - shadowed by $(x, v) \in \Lambda$ if $d\left(\varphi_{g}^{t}(x, v), P(t)\right)<\varepsilon$ for all $t \in \bigcup_{I_{i} \in \sigma} I_{i}$.

We say that $\Lambda$ has the weak specification property if for any $\varepsilon>0$ there exists a $K=K(\varepsilon) \in \mathbb{R}$ such that any $K$-spaced weak specification $\mathcal{S}$ is $\varepsilon$-shadowed by a point of $\Lambda$. In this case $\left.\varphi_{g}^{t}\right|_{\Lambda}$ is said to have the weak specification property. The geodesic flow of $g$ is said to have the weak specification property if $S_{g} M$ has it.

The geodesic flow has the stable weak specification property if there exists a $C^{2}$-neighbourhood $\mathcal{V} \subset \mathcal{R}^{\infty}$ of $g$ such that $\varphi_{\hat{g}}^{t}$ for any $\hat{g} \in \mathcal{V}$ has the weak specification property.

2.7. The topological stability property. Consider the geodesic flows $\varphi_{g}^{t}: S_{g} M \rightarrow S_{g} M$ and $\varphi_{\tilde{g}}^{t}: S_{\tilde{g}} M \rightarrow S_{\tilde{g}} M$ associated to the metrics $g, \tilde{g} \in \mathcal{R}^{\infty}$, respectively. We say that $\tilde{g}$ is semiconjugated to $g$ if there exist a continuous and surjective map $h: S_{\tilde{g}} M \rightarrow S_{g} M$ and a continuous real map $\tau: S_{\tilde{g}} M \times \mathbb{R} \rightarrow \mathbb{R}$ such that

(a) for any $(x, v) \in S_{\tilde{g}} M, \tau_{(x, v)}: \mathbb{R} \rightarrow \mathbb{R}$ is an orientation preserving homeomorphism where $\tau((x, v), 0)=0$ and

(b) for all $(x, v) \in S_{\tilde{g}} M$ and $t \in \mathbb{R}$ we have $h\left(\varphi_{\tilde{g}}^{t}(x, v)\right)=\varphi_{g}^{\tau((x, v), t)}(h(x, v))$.

We say that the geodesic flow of $g$ is topologically stable if for any $\epsilon>0$ there exists $\delta>0$ such that for any metric $\tilde{g}$ such that $\tilde{g}$ is $\delta$-C $C^{1}$-close to $g$ there exists a semiconjugacy from $S_{\tilde{g}} M$ to $S_{g} M$, i.e. there exists $h: S_{\tilde{g}} M \rightarrow S_{g} M$ and $\tau: S_{\tilde{g}} M \times \mathbb{R} \rightarrow \mathbb{R}$ satisfying (a) and (b) above, and

$$
d(h(x, v),(x, v))<\epsilon, \quad(x, v) \in S_{\tilde{g}} M .
$$

Notice that the notion of topologically stability does not define an equivalence relation. Furthermore, the set of systems semi-conjugated to a given metric might not be an open set. This motivates the following definition: $g$ is $C^{2}$-robustly topologically stable if there exists a $C^{2}$-neighbourhood $\mathcal{V} \subset \mathcal{R}^{\infty}$ of $g$ whose elements have topologically stable geodesic flows.

2.8. The Kupka-Smale theorem revisited. A proof of the Kupka-Smale theorem for geodesic flows is included in [9, section 2] (notice that the statement there differs from the one presented in [13]). We explain below how to obtain a sharper version of the theorem to be used in the sequel.

Let $J_{s}^{r}(2 n-2)$ be the set of $r$-jets of symplectic automorphisms of $\mathbb{R}^{2 n-2}$ which fix the origin and $r \geq 1$. One can identify $J_{s}^{1}(2 n-2)$ with $\operatorname{Sp}(2 n-2, \mathbb{R})$. A set $Q \subset J_{s}^{r}(2 n-2)$ is said to be invariant if for all $\sigma \in J_{s}^{r}(2 n-2), \sigma Q \sigma^{-1}=Q$.

Theorem 2.6. (Kupka-Smale [11,9]) Let $Q \subset J_{s}^{r}(2 n-2)$ be open, dense and invariant. Then, there exists a residual subset $\mathcal{K} \mathcal{S} \subset \mathcal{R}^{r+1}$ such that for all $g \in \mathcal{K} \mathcal{S}$ we have: 
(i) The $r$-jet of the Poincaré map of every closed geodesic of $g$ belongs to $Q$;

(ii) All heteroclinic points of hyperbolic closed geodesics of $g$ are transversal.

The proof of (ii) of Theorem 2.6 is in [9] and it is based on the construction of a perturbation [9, Lemma 2.6] that guarantees the transversality. One first obtains a Hamiltonian local perturbation of the geodesic flow with the transversality property (see [13]) related to a perturbed unstable Lagrangian manifold. A perturbed metric is then constructed such that this manifold is inside the energy level set. As it is Lagrangian it is invariant and coincides with the unstable manifold elsewhere for the geodesic flow. This means that the new metric has the transversality property.

Theorem 2.7. Under the conditions of Theorem 2.6, there are no heteroclinic points for any pair of periodic points consisting in a hyperbolic point and a 1-elliptic point.

Proof. We follow the arguments in the proof of Theorem 2.5 in [9], using the same notation, to obtain the empty intersection property. Let $\mathcal{H}_{N}^{r+1}$ be the set of $C^{r+1}$ Riemannian metrics $g$ such that the $r$-jet of the Poincaré map of every closed orbit of $g$ with period less or equal than $N$ belongs to $Q$ (with 1-jets partially hyperbolic, hyperbolic or totally elliptic). By [9, Corollary 2.2] generically there are only a finite number of them. Let $\mathcal{K}_{N}^{r+1}$ be the subset of $\mathcal{H}_{N}^{r+1}$ of those metrics $g$ such that for any pair of:

(i) hyperbolic closed orbits $\gamma$ and $\eta$ of $g$ with period less or equal than $N$, the submanifolds $W_{N}^{s}(\gamma)$ an $W_{N}^{u}(\eta)$ are transversal, where $W_{N}^{s}(\gamma)$ is given by those points $\theta \in W^{s}(\gamma)$ with $d_{W^{s}(\gamma)}(\theta, \gamma)<N$ (analogous definition for $W_{N}^{u}(\eta)$ );

(ii) periodic points consisting in a hyperbolic point $\eta$ and a 1-elliptic point $\gamma$ of $g$ with period less or equal than $N$, the submanifolds $W_{N}^{u}(\eta)$ an $W_{N}^{s}(\gamma)$ do not intersect (analogously, we can switch the role of the stable/unstable manifolds).

Since the stable and unstable manifolds of a closed orbit depend continuously on compact parts in the $C^{1}$ topology we conclude that $\mathcal{K}_{N}^{r+1}$ is an open subset of $\mathcal{R}^{r+1}$. Meaning that, in case (i), we have persistence of transversal intersection and, in case (ii), we have persistence of empty intersection of stable/unstable manifolds. If we prove that $\mathcal{K}_{N}^{r+1}$ is a dense subset of the $C^{r+1}$ metrics, then the residual subset of the theorem is defined by $\bigcap_{N \in \mathbb{N}} \mathcal{K}_{N}^{r+1}$.

In [9] it was proved that the set defined as our set $\mathcal{K}_{N}^{r+1}$ but only satisfying (i) is dense in the $C^{r+1}$ metrics. Now, we show that our 'smaller' subset $\mathcal{K}_{N}^{r+1}$ is also dense. To do that, we first observe that $C^{r+1}$-generically (hence, by Baire's category theorem, $C^{r+1}$-densely) there are only a finite number of partially hyperbolic closed orbits with period less or equal than $N$. Therefore our task of perturbing the metric to unstick non-transversal intersections of stable/unstable manifolds of a pair hyperbolic/1-elliptic closed orbits can be achieved performing a finite number of perturbations.

Lemma 2.8. $\mathcal{K}_{N}^{r+1}$ is dense in $\mathcal{R}^{r+1}$.

Proof. We begin by assuming that the geodesic flow of $g$ has only a finite number of partially hyperbolic closed orbits of period less or equal than $N$. If there exist partially hyperbolic closed orbits $\gamma$ and $\eta$ of $g$ with period less or equal than $N$ such that the submanifolds $W_{N}^{s}(\gamma)$ and $W_{N}^{u}(\eta)$ do not intersect, then they will never intersect.

Now, perturb locally the metric in order to have $W_{N}^{u}(\eta)$ non-intersecting (i.e. transversal to) the initial $W_{N}^{s}(\gamma)$ in a small open subset intersecting a (compact) fundamental domain $K$ of $W^{u}(\eta)$.

It can be used the same idea mentioned right after Theorem 2.6. Firstly one finds a local Hamiltonian perturbation yielding an empty intersection between the Lagrangian unstable manifold of the hyperbolic closed orbit and the isotropic stable manifold of the 1-elliptic closed orbit. 
Indeed, $W^{u}(\eta)$ and $W^{s}(\gamma)$ can become disjoint using a perturbation along the 2-dimensional central manifold $W^{c}(\gamma)$. The realization of this effect for a perturbed metric is again obtained as in [9, Lemma 2.6].

Cover $K$ with a finite number of those small open subsets and unstick all intersections. Finally, we observe two facts: a) our perturbations will cause a change in the new stable/unstable manifolds but do not change the future of $W^{s}(\gamma)$ neither the past of $W^{u}(\eta)$; b) once all intersections are removed in a fundamental domain it will spread through the whole manifold.

Finally, we obtain that all intersections are removed under arbitrarily small perturbation on the metric.

This completes the proof of Theorem 2.7.

\section{Proofs}

3.1. Proof of Theorem 1 (A). Assume that there is a $q$-elliptic closed orbit $(q \geq 2)$.

Following Lemma 2.2, there is nearby a 1-elliptic closed orbit. The Poincaré map restricted to the 2-dimensional invariant central manifold $W^{s c}$ of this 1-elliptic closed orbit is an areapreserving twist map.

Consider a pseudo-orbit constructed in $W^{s c}$ and assume that the eigenvalues of the linearized Poincaré map restricted to $W^{s c}$ are equal to $e^{ \pm i \rho}$ with $\rho$ irrational. Then, by a straighforward adaptation of the proof of [6, Proposition 4.1(a)], there can not exist a shadowing orbit in $W^{s c}$. So, there is $\varepsilon>0$ such that for any $\delta, T>0$ we can find a $(\delta, T)$-pseudo-geodesic $\left[\left(x_{n}, v_{n}\right),\left(t_{n}\right)\right]_{n \in \mathbb{N}}$ which is not $\varepsilon$-shadowed by any orbit of $g$ in $W^{s c}$.

It remains to consider the case that the shadowing orbit is outside $W^{s c}$. Since the central manifold is normally hyperbolic, the unstable directions will drive away the orbit from an $\varepsilon$ neighbourhood of the pseudo-orbit, thus shadowing with reparametrization near the identity is not possible.

By restricting the metric $g$ to the residual set $\mathcal{G}_{1} \cap \mathcal{R}^{\infty}$, we can use Lemma 2.4 in order to perturb $g$ and get $\rho$ irrational for the new metric. Thus, the shadowing properties can not stably hold.

3.2. Proof of Theorem 1 (B). Several authors obtained hyperbolicity from the hypothesis that the system has the stable specification property (see $[16,2]$ ). We point out that their arguments were supported on a change of index argument in the hyperbolic closed orbits. However, in the symplectic setting such situation is impossible to happen because the index is constant and equal to $n-1$. In [5] this problem was solved using a pasting lemma available for Hamiltonians but not available for the geodesic flow. Here, we obtain similar results for geodesic flows through a new approach that we explain in the following.

Notice first that given two $q$-elliptic closed orbits, $1 \leq q \leq n-2$, and under the weak specification property we must have non-empty intersection between the stable manifold of one with the unstable manifold of the other. Clearly, this intersection cannot be transversal. Therefore, considering solely Kupka-Smale geodesic flows (see §2.8) with the weak specification property, we conclude that $q$-elliptic closed orbits cannot exist. Consider then the case of a totally elliptic closed orbit (i.e. $q=n-1$ ) on geodesic flows with robust weak specification. But this implies by Lemma 2.2 the existence of a 1-elliptic closed orbit. So, under stability of weak specification the periodic points can only be hyperbolic.

We shall start by deducing some consequences of the weak specification property. Let $g \in \mathcal{R}^{\infty}$ and $\gamma_{0}=(x, v) \in S_{g} M$ be a periodic point. Recall that we denote the spectrum of $D P_{g}\left(\gamma_{0}\right)$ outside the unit circle by $\mathbb{S}^{0}\left(g, \gamma_{0}\right)$ and by $\gamma$ the orbit of the point $\gamma_{0}$.

The next result is an adaptation of [2, Theorem 3.3]. 
Lemma 3.1. Let $g \in \mathcal{R}^{\infty}$. If $\varphi_{g}^{t}$ has the weak specification property, then for every distinct periodic points $\gamma_{0}, \eta_{0} \in S_{g} M$ such that $\mathbb{S}^{0}\left(g, \gamma_{0}\right) \neq \emptyset$ and $\mathbb{S}^{0}\left(g, \eta_{0}\right) \neq \emptyset$, we have that

$$
W^{u}(\gamma) \cap W^{s}(\eta) \neq \emptyset .
$$

Proof. Denote by $\epsilon\left(\gamma_{0}\right)$ the size of the local strong unstable manifold $W_{\epsilon\left(\gamma_{0}\right)}^{s u}\left(\gamma_{0}\right)$ of $\gamma_{0}$ and by $\epsilon\left(\eta_{0}\right)$ the size of the local strong stable manifold $W_{\epsilon\left(\eta_{0}\right)}^{s s}\left(\eta_{0}\right)$ of $\eta_{0}$. Let $\epsilon=\min \left\{\epsilon\left(\gamma_{0}\right), \epsilon\left(\eta_{0}\right)\right\}$ and $K=K(\epsilon)$ be given by the weak specification property. If $t>0$, take $I_{1}=[0, t]$ and $I_{2}=[K+t, K+2 t]$.

Now, define $P(s)=\varphi_{g}^{s-t}\left(\gamma_{0}\right)$ if $s \in I_{1}$ and $P(s)=\varphi_{g}^{s-K-t}\left(\eta_{0}\right)$ if $s \in I_{2}$. Note that this is a $K$-spaced weak specification. Under these conditions there exists $\zeta_{0}^{t}$ which shadows this weak specification,

$$
d\left(\varphi_{g}^{s}\left(\zeta_{0}^{t}\right), P(s)\right) \leq \epsilon \text { if } s \in I_{1} \cup I_{2} .
$$

Using the change of variable $u=t-s$, we have

$$
d\left(\varphi_{g}^{-u}\left(\varphi_{g}^{t}\left(\zeta_{0}^{t}\right)\right), \varphi_{g}^{-u}\left(\gamma_{0}\right)\right)=d\left(\varphi_{g}^{t-u}\left(\zeta_{0}^{t}\right), \varphi_{g}^{-u}\left(\gamma_{0}\right)\right) \leq \epsilon, \quad u \in[0, t]
$$

and with $u=s-K-t$,

$$
d\left(\varphi_{g}^{u}\left(\varphi_{g}^{K+t}\left(\zeta_{0}^{t}\right)\right), \varphi_{g}^{u}\left(\eta_{0}\right)\right) \leq \epsilon, \quad u \in[0, t] .
$$

If $\xi_{0}^{t}=\varphi_{g}^{t}\left(\zeta_{0}^{t}\right)$ we can assume that $\xi_{0}^{t} \rightarrow \xi_{0}$. By taking limits in the previous inequalities we obtain

$$
\begin{gathered}
d\left(\varphi_{g}^{-u}\left(\xi_{0}\right), \varphi_{g}^{-u}\left(\gamma_{0}\right)\right) \leq \epsilon \text { for every } u \geq 0, \text { and } \\
d\left(\varphi_{g}^{u}\left(\varphi_{g}^{K}\left(\xi_{0}\right)\right), \varphi_{g}^{u}\left(\eta_{0}\right)\right) \leq \epsilon \text { for every } u \geq 0 .
\end{gathered}
$$

The first one says that $\xi_{0} \in W_{\epsilon}^{s u}\left(\gamma_{0}\right) \subset W^{u}(\gamma)$ and the second one says that $\varphi_{g}^{K}\left(\xi_{0}\right) \in W_{\epsilon}^{s s}\left(\eta_{0}\right)$, hence $\xi_{0} \in W^{s}(\eta)$.

The proof of the next result differs substantially from the one in [5] for Hamiltonians.

Proposition 3.2. Let $g \in \mathcal{R}^{\infty}$. If $\varphi_{g}^{t}$ has the stable weak specification property, then $\mathbb{S}^{0}\left(g, \gamma_{0}\right) \neq$ $\emptyset$ for any periodic point $\gamma_{0}$.

Proof. Let $\mathcal{V}$ be a $C^{2}$-neighbourhood of $g$ wherein the weak specification property holds. Suppose, by contradiction, that there exists a totally elliptic periodic point $\gamma_{0}$ of $\varphi_{g}^{t}$. We can approximate the metric $g$ by $\tilde{g} \in \mathcal{G}_{0} \cap \mathcal{V}$ so that the analytic continuation of the totally elliptic periodic point $\gamma_{0}$ remains a totally elliptic periodic point. Now, using Lemma 2.2 , we obtain a 1-elliptic periodic point $\tilde{\gamma_{0}}$ near $\gamma_{0}$. The case $n=2$ is contained in [6, Theorem 1 (c)].

Assume that $n>2$. By $\left[8\right.$, Theorem A] we obtain a nontrivial hyperbolic basic set $\Lambda_{g_{1}}$ for a metric $g_{1} \in \mathcal{V}$ such that we still have a continuation of the 1-elliptic periodic orbit $\tilde{\gamma}$ (also denoted by $\tilde{\gamma}$ ). Further perturbing using the Kupka-Smale theorem (Theorem 2.6) we get $g_{2} \in \mathcal{K} \mathcal{S} \cap \mathcal{V}$ yelding a continuation of the 1-elliptic periodic orbit $\tilde{\gamma}$ (again denoted by $\tilde{\gamma}$ ) and a continuation $\Lambda_{g_{2}}$ of the nontrivial hyperbolic basic set.

Let $\eta_{0} \in \Lambda_{g_{2}}$ be a hyperbolic periodic point and so $\operatorname{dim} W^{s}(\eta)=n$. As $\operatorname{dim}\left(S_{g} M\right)=2 n-1$ and since $\tilde{\gamma}_{0}$ is a 1-elliptic periodic point, we have that $\operatorname{dim} W^{u}(\tilde{\gamma})=\operatorname{dim} W^{s}(\tilde{\gamma})=n-1$. To obtain a transversal intersection between $W^{u}(\tilde{\gamma})$ and $W^{s}(\eta)$ one must have

$$
\operatorname{dim}\left[W^{u}(\tilde{\gamma}) \cap W^{s}(\eta)\right]=\operatorname{dim} W^{u}(\tilde{\gamma})+\operatorname{dim} W^{s}(\eta)-\operatorname{dim}\left(S_{g} M\right),
$$

which can never happen in our context. Indeed, since the stable/unstable manifolds intersect transversally (cf. Theorem 2.6 and Theorem 2.7), one has $W^{u}(\tilde{\gamma}) \cap W^{s}(\eta)=\emptyset$. But this contradicts Lemma 3.1. 
The previous proof easily entails the following result.

Lemma 3.3. Let $g \in \mathcal{R}^{\infty}$ such that $\varphi_{g}^{t}$ has the stable weak specification property for a neighbourhood $\mathcal{V}$ of $g$. Then, every Kupka-Smale metric in $\mathcal{V}$ has all closed orbits of hyperbolic type.

The lemma below is a direct consequence of Theorem 2.6.

Lemma 3.4. Let $g \in \mathcal{R}^{\infty}$ and let $\gamma$ be a non-hyperbolic closed orbit of $\varphi_{g}^{t}$. Then, there exists $\tilde{g} \in \mathcal{R}^{\infty}$ arbitrarily close to $g$ such that $\tilde{g}$ is a Kupka-Smale metric exhibiting elliptic closed orbits.

Proof. Let $\gamma$ be a non-hyperbolic closed orbit of $\varphi_{g}^{t}$. If $\gamma$ is $q$-elliptic with $1 \leq q \leq n-1$, there exists an arbitrarily $C^{2}$-close Kupka-Smale metric $\tilde{g} \in \mathcal{K} \mathcal{S}$ such that the analytic continuation of the elliptic closed orbit $\gamma$ is still a $q$-elliptic closed orbit for the Kupka-Smale metric $\tilde{g}$.

We can now prove Theorem 1 (B). Given a metric $g \in \mathcal{R}^{\infty}$ satisfying the stable weak specification property on a neighborhood $\mathcal{V}$, we need to show that all its closed orbits are hyperbolic. Hence, $g \in \mathscr{F}^{2}$. Suppose that there is a non-hyperbolic closed orbit $\gamma$. By Lemma 3.4 there exists a Kupka-Smale metric $\tilde{g} \in \mathcal{V}$ such that $\varphi_{\tilde{g}}^{t}$ has a non-hyperbolic closed orbit, which contradicts Lemma 3.3 .

3.3. Proof of Theorem 1 (C). Let $L>0$ and fix $\eta>0$ such that any $\hat{g} \in \mathcal{R}^{\infty}$ being $\eta$ - $C^{2}$ close to $g$ still has the shadowing property with reparametrization $\tau \in \operatorname{Rep}^{\prime}(L)$. Take any closed orbit $\gamma$ of $\varphi_{g}^{t}$ and suppose, by contradiction, that $\gamma$ is $q$-elliptic $(q \geq 2)$. By Lemma 2.2 there exists a 1-elliptic closed orbit $\tilde{\gamma}$ near $\gamma$ whose Poincaré map $P_{g}$ on the 2-dimensional central manifold $W^{s c}$ is an area-preserving twist map.

As in the proof of Theorem 1 (A), for any $\delta>0$ we will consider a $\delta$-pseudo-orbit constructed in $W^{s c}$ and assume that the eigenvalues of the linearized Poincaré map restricted to $W^{s c}$ are equal to $e^{ \pm i \rho}$ with $\rho$ irrational.

Let $\sigma>1$ be the largest eigenvalue of $D P_{g}$. Take $\tilde{\gamma}_{0}=\tilde{\gamma} \cap W^{s c}$ and $\epsilon<\hat{\epsilon} \sigma^{-L}$, where $\hat{\epsilon}>0$ is the radius of a domain of linearization of $P_{g}$ in a neighborhood of $\tilde{\gamma}_{0}$. The true orbit which $\epsilon$-shadows the $\delta$-pseudo-orbit cannot be in the central manifold, so it must have hyperbolic expansive components but surely dominated by $\sigma$. Considering neighborhoods of radius $\epsilon$ and $\sigma^{L} \epsilon$ from the 1-elliptic closed orbit we know that the true orbit will remain in the region between $\epsilon$ and $\sigma^{L} \epsilon<\hat{\epsilon}$ for at least $\lfloor L\rfloor$ iterates. This contradicts the fact that $g$ has the shadowing property with reparametrization $\tau \in \operatorname{Rep}^{\prime}(L)$.

\section{ACKNOWLEDGEMENTS}

The authors would like to thank the anonymous referee for the careful reading of the manuscript and for giving helpful comments and suggestions.

The authors were partially supported by the Project 'New trends in Lyapunov exponents' (PTDC/MAT-PUR/29126/2017).

MB was partially supported by FCT - 'Fundação para a Ciência e a Tecnologia', through Centro de Matemática e Aplicações (CMA-UBI), Universidade da Beira Interior, project UID/MAT/00212/2013. MB also like to thank CMUP for providing the necessary conditions in which this work was developed.

JLD was partially supported by the Project CEMAPRE - UID/MULTI/00491/2019 financed by FCT/MCTES through national funds.

MJT was partially supported by the Research Centre of Mathematics of the University of Minho with the Portuguese Funds from the Fundação para a Ciência e a Tecnologia, through the Project UID/MAT/00013/2013. 


\section{REFERENCES}

[1] R. Abraham, Bumpy metrics, Global Analysis, Proc. Sympos. Pure Math., XIV (S.S. Chern and S. Smale, eds.), 1970, 1-3.

[2] A. Arbieto, L. Senos, and T. Sodero, The specification property for flows from the robust and generic view point, J. Differential Equations, 253 (6) (2012), 1893-1909.

[3] M.-C. Arnaud, Type des Points Fixes des Difféomorphismes Symplectiques de $\mathbb{T}^{n} \times \mathbb{R}^{n}$, Mém. Soc. Math. France 48, 1992.

[4] M. Bessa, J. Rocha, On $C^{1}$-robust transitivity of volume-preserving flows, J. Differential Equations, 245 (11) (2008), 3127-3143.

[5] M. Bessa, J. Rocha and M. J. Torres, Shades of hyperbolicity for Hamiltonians, Nonlinearity, 26 (10) (2013), $2851-2873$.

[6] M. Bessa, J. Lopes Dias and M. J. Torres, On shadowing and hyperbolicity for geodesic flows on surfaces, Nonlinear Anal., 55 (2017), 250-263.

[7] R. Bowen, Periodic points and measures for Axiom A diffeomorphisms, Trans. Amer. Math. Soc., 154 (1971), 37-397.

[8] G. Contreras, Geodesic flows with positive topological entropy, twist maps and hyperbolicity, Annals Math., 172 (2010), 761-808.

[9] G. Contreras and G. Paternain, Genericity of geodesic flows with positive topological entropy on $S^{2}$, J. Differential Geom., 61 (1) (2002), 1-49.

[10] M. Denker, C. Grillenberger and K. Sigmund, Ergodic Theory on Compact Spaces, Lecture Notes in Math. 527 (Springer-Verlag, Berlin, 1976).

[11] W. Klingenberg and F. Takens, Generic properties of geodesic flows, Math. Ann., 197 (1972), 323-334.

[12] L. Rifford, Closing geodesics in $C^{1}$ topology, J. Differential Geom., 91 (2012), 361-381.

[13] C. Robinson, Generic properties of conservative systems, Amer. J. Math., 92 (1970), 562-603 and 897-906.

[14] R. Ruggiero, Persistently expansive geodesic flows, Comm. Math. Phys., 140 (1) (1991), 203-215.

[15] D. Visscher, A new proof of Franks' lemma for geodesic flows, Discrete and Continuous Dynamical SystemsSeries A, 34 (2014), 4875-4895.

[16] K. Sakai, N. Sumi and K. Yamamoto, Diffeomorphisms satisfying the specification property, Proc. Amer. Math. Soc., 138 (1) (2010), 315-321.

[17] P. Walters, On the pseudo-orbit tracing property and its relationship to stability. in 'Dynamical SystemsWarwick 1974' (A. Manning. Ed.), pp. 231-244. Springer Lecture Notes No. 468, Springer-Verlag, New York/Berlin. 1975.

Universidade da Beira Interior, Rua Marquês d’ávila e Bolama, 6201-001 Covilhã, Portugal Email address: bessa@ubi.pt

Departamento de Matemática, Cemapre and REM, ISEG, Universidade de Lisboa, Rua do Quelhas 6, 1200-781 Lisboa, Portugal

Email address: jldias@iseg.ulisboa.pt

Cmat and Departamento de Matemática e AplicaÇões, Universidade do Minho, Campus de GualTAR, 4700-057 Braga, Portugal

Email address: jtorres@math.uminho.pt 